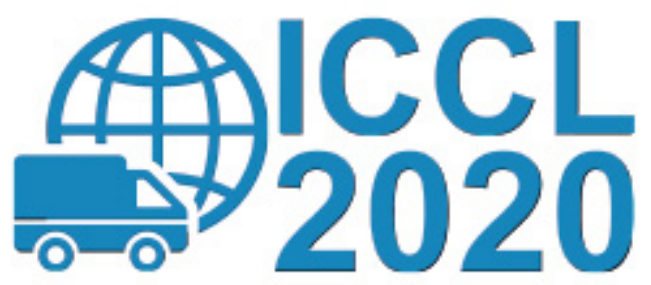

$11^{\text {th }}$ International Conference on Computational Logistics

September 28-30, 2020,

hosted by the University of Twente,

Enschede, The Netherlands.

\author{
held online
}

BOOK OF ABSTRACTS 


\title{
Solving a Bi-Objective Rich Vehicle Routing Problem with Customer Prioritization
}

\author{
Tim van Benthem ${ }^{1}$, Mark Bergman ${ }^{1}$, Martijn Mes $^{1}$ \\ ${ }^{1}$ University of Twente, the Netherlands \\ t.s.vanbenthemestudent.utwente.nl
}

\begin{abstract}
This paper considers a rich vehicle routing problem in which a combination of transportation costs and customerperceived waiting times should be minimized and a differentiation is made between priority and non-priority customers. We illustrate the problem using a case study of a wholesaler with its own lastmile delivery network where customers can have pickup and delivery demand and are served by a heterogeneous fleet of vehicles. We propose a bi-objective mathematical problem formulation, minimizing the combination of transportation costs and customer dissatisfaction. We model customer dissatisfaction using a non-linear function that approximates the perceived waiting time of the customers. To be able to solve realisticallysized problems in reasonable time, we propose a Simulated Annealing heuristic, Variable Neighborhood Search, and a combination of these. We perform various experiments considering different customer preferences (visit as soon as possible or at a specific time) and problem settings. For the combined objective, we see an average costs reduction for the dissatisfaction function approach compared to the standard time window approach of $48 \%$ over all experiments. Furthermore, we observe an average reduction in perceived waiting time of $48 \%$ and $20 \%$ for priority and non-priority customers, respectively.
\end{abstract}

Keywords: Vehicle Routing Problem, Customer Satisfaction, Simulated Annealing, Variable Neighborhood Search, Time Windows 